\title{
ASYMPTOTIC ANALYSIS OF A TRANSLATING CABLE ARCH
}

\author{
N. C. Perkins \\ Mechanical Engineering and Applied Mechanics, University of Michigan, Ann Arbor, \\ Michigan 48109, U.S.A.
}

(Received 25 July 1988, and in revised form 17 March 1989)

\begin{abstract}
The stability of a translating cable which stands in the shape of an arch between two supports is investigated. Asymptotic solutions for free in-plane and out-of-plane cable vibrations are derived under the assumption of small arch height. Without translation speed, the cable arch collapses under compressional loading. As translation speed is increased, however, the cable arch becomes tensioned and stable. A previous experiment confirms this prediction. The asymptotic solutions derived here show that the in-plane and out-of-plane vibration modes are stabilized in fundamentally different manners.
\end{abstract}

\section{INTRODUCTION}

The motion of a string translating between two eyelets is the archetypical problem in the dynamics of axially moving materials [1]. The translating string, commonly referred to as the moving threadline [2], has served as a useful model for textile machinery, chain and belt drives, fiber winders and magnetic tape drives. These diverse applications and others have been discussed by Wickert and Mote [3], who presented a detailed summary of current research in the vibration and stability of axially moving materials.

The present investigation is concerned with the dynamic stability of a translating and sagged cable which is used to model the cable-like elements found in overhead conveyor systems, ski lifts, aerial tramways, and slack belt drives. Initial curvature creates linear coupling of longitudinal and transverse motions in the plane of the cable equilibrium. This linear in-plane motion is decoupled from the linear out-of-plane motion. Simpson [4] analyzed the free linear in-plane vibration of a translating cable using an asymptotic solution valid for cables with small sag $\dagger$ and level support eyelets. Later, Triantafyllou [5] extended the asymptotic solution to include cables having inclined supports and either small or very large sag. The numerical analysis by Perkins and Mote [6] considered complete three-dimensional cable motions and allowed arbitrary cable sag and support inclination. Due to initial cable curvature, the equilibrium cable tension increases with translation speed $[4,6]$ and the translating cable can never experience the buckling instability of the simpler moving threadline [2]. An experimental investigation of a translating cable is included in a recent paper by Perkins and Mote [7] and confirms the theoretical findings of reference [6].

These investigations of translating cables have been concerned with the free linear in-plane and out-of-plane vibration of an elastic cable about an equilibrium which hangs between the supports under the influence of gravity. This equilibrium is termed the minimum catenary. In reference [7], a second equilibrium, referred to as the maximum catenary, was noted: i.e., a cable which stands in the shape of an arch between the supports. Without translation speed, the maximum catenary collapses under compressional loading. The maximum catenary, however, becomes tensioned with increasing

† Small sag is defined by a cable sag to span ratio of $\frac{1}{8}$ or less. 
translation speed and is eventually stabilized as evidenced by the numerical analysis and experiment of reference [7]. The experiment clearly demonstrates that, while rare in most applications, a stable maximum catenary equilibrium may exist under very high speed conditions.

This paper re-examines the stability of the maximum catenary for the case of a "shallow cable arch". An asymptotic solution for free linear three-dimensional vibration is derived and confirms the purely numerical solution obtained in reference [7]. The out-of-plane and the in-plane vibration modes are observed to become stabilized in fundamentally different ways. The eigenvalues governing the stability of out-of-plane modes change from imaginary to real values through a singularity at a critical cable translation speed. By contrast, the eigenvalues associated with the in-plane modes assume complex values during their transition from imaginary to real values.

\section{EQUATIONS OF MOTION}

A summary of the theoretical model for a translating elastic cable is provided here and follows from the complete derivation in reference [6]. The equations of motion and the solution to the cable equilibrium problem are derived with reference to Figure 1 which depicts an elastic cable translating with speed $v_{c}$, between two level support eyelets.

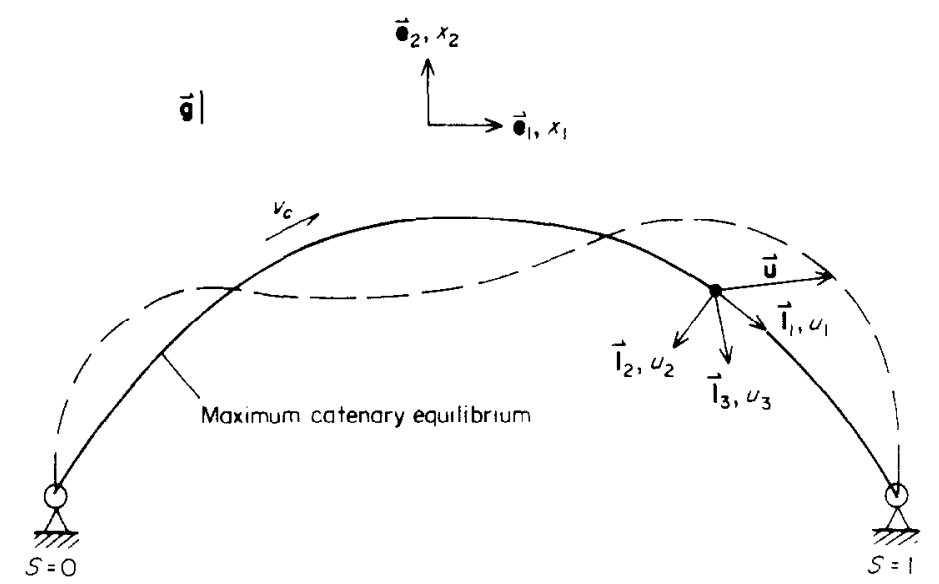

Figure 1. The arch-like maximum catenary equilibrium is formed by a cable which translates with speed $v_{c}$. between two fixed support eyelets. $U$ represents motion from the cable equilibrium and is decomposed into components aligned with the Frenet triad $\left(I_{1}, I_{2}, I_{3}\right)$.

Analysis of the cable equilibrium problem [7] reveals that there are two possible equilibria referred to as the minimum catenary and the maximum catenary. The minimum catenary describes a cable which sags between the supports [4-6]. In this study, attention is focused on the maximum catenary of Figure 1 which stands in the shape of an arch between the level supports. The maximum catenary lies in the vertical plane with gravity $\mathbf{g}$ aligned with the $\mathbf{e}_{2}$ direction and is given by the co-ordinates [7]:

$$
x_{2}\left(x_{1}\right)=m^{2}\left[\cosh \left(x_{1} / m^{2}\right)\right]
$$

The constant $m^{2}$ is

$$
m^{2}=v_{1}^{2}-v_{c}^{2}
$$

where

$$
v_{i}^{2}=P_{0} / \rho g A^{i}, \quad v_{c}^{2}=\rho A^{i} c^{i^{2}} / \rho g A^{i}
$$


Here, $P_{0}$ is the cable tension (or compression) at the mid-span $x_{1}=0, \rho$ is the cable mass/length, $A^{i}$ is the cable cross-sectional area, $L^{i}$ is the cable length and $c^{i}$ is the cable translation speed. As defined by equation (2), the constant $m^{2}$ is positive for the minimum catenary and negative for the maximum catenary [7], and for convenience in this study, the positive constant

$$
n^{2}=v_{c}^{2}-v_{t}^{2}
$$

is introduced. The equilibrium problem also provides the initial cable tension $v_{t}^{2} p$ and curvature $k$ :

$$
\begin{gathered}
v_{l}^{2} p(s)=v_{c}^{2}-\left[\left(n^{2}\right)^{2}+\left(s-\frac{1}{2}\right)^{2}\right]^{1 / 2}, \\
k(s)=-n^{2} /\left[\left(n^{2}\right)^{2}+\left(s-\frac{1}{2}\right)^{2}\right] .
\end{gathered}
$$

Here $s$ is the non-dimensional cable arc length co-ordinate which is related to the dimensional co-ordinate $S^{i}$ through

$$
s=\left(S^{i} / L^{i}\right)+\frac{1}{2}
$$

Note from equation (6) that the cable is in compression $\left(v_{t}^{2} p<0\right)$ when the translation speed vanishes $\left(v_{c}^{2}=0\right)$ but may become tensioned $\left(v_{t}^{2} p>0\right)$ for sufficiently large translation speed. This speed tensioning capability of the cable equilibrium allows the maximum catenary to be stabilized [7].

The vector $\mathbf{u}(s, t)$ in Figure 1, representing the three-dimensional motion of the cable about its equilibrium, is resolved into the components $\left(u_{1}, u_{2}, u_{3}\right)$ aligned with the local tangential $l_{1}$, normal $l_{2}$, and bi-normal $\mathbf{l}_{3}$ directions of the cable equilibrium. These components are governed by the equations of motion derived in reference [7] which, after linearization about the maximum catenary, become as follows:

tangential component, $u_{1}$,

$$
\begin{aligned}
& {\left[\begin{array}{l}
\left.v_{l}^{2}\left(u_{1, s}-k u_{2}\right)\right]_{, s}-k\left[\left(v_{t}^{2} p\right)\left(u_{2, s}+k u_{1}\right)\right] \\
\quad=u_{1, t t}+2 v_{c}\left(u_{1, s}-k u_{2}\right)_{, t}+v_{c}^{2}\left[\left(u_{1, s}-k u_{2}\right)_{, s}-k\left(u_{2, s}+k u_{1}\right)\right]
\end{array}\right.}
\end{aligned}
$$

normal component, $u_{2}$,

$$
\begin{aligned}
& {\left[\left(v_{1}^{2} p\right)\left(u_{2, s}+k u_{1}\right)\right]_{, s}+k\left[\left(v_{l}^{2}\right)\left(u_{1, s}-k u_{2}\right)\right]} \\
& \quad=u_{2, t}+2 v_{c}\left(u_{2, s}+k u_{1}\right)_{, t}+v_{c}^{2}\left[\left(u_{2, s}+k u_{1}\right)_{, s}+k\left(u_{1, s}-k u_{2}\right)\right]
\end{aligned}
$$

bi-normal component, $u_{3}$,

$$
\left[\left(v_{t}^{2} p\right)\left(u_{3, s}\right)\right]_{, s}=u_{3, t}+2 v_{c} u_{3, s t}+v_{c}^{2} u_{3, s s}
$$

boundary conditions

$$
u_{j}(0, t)=u_{j}(1, t)=0 \quad \text { for } j=1,2,3 .
$$

The remaining non-dimensional quantities appearing in equations (9-12) are

$$
t=T\left(g / L^{i}\right)^{1 / 2}, \quad u_{j}=U_{j} / L^{i}, \quad j=1,2,3, \quad v_{l}^{2}=E A^{i} /\left(\rho g A^{i} L^{i}\right),
$$

where $T, U_{j}$, and $E$ denote dimensional time, displacement and Young's modulus, respectively.

General analytical solutions have not yet been found for the coupled equations $(9,10)$ describing free in-plane motion and the uncoupled equation (11) which governs free out-of-plane motion. Asymptotic solutions have been derived for special cases of the related minimum catenary problem $[4,5]$ by employing assumptions which, in essence, simplify the form of the non-constant coefficients $v_{t}^{2} p(s)$ and $k(s)$ appearing in these equations of motion. In a previous study [7], the Galerkin method was employed to obtain approximate solutions for the natural frequencies and mode shapes for free vibration of the maximum catenary. 
2.1. APPROXIMATE THEORY FOR SHALLOW CABLE ARCH

An asymptotic solution for the maximum catenary problem is obtained by restricting attention to maximum catenaries having small arch height. For such catenaries, the equilibrium tension (6) is nearly constant and the curvature (7) is small. For $n^{2} \gg !$, first order approximations to equation (6) and (7) are

$$
v_{t}^{2} p(s)=v_{c}^{2}-\left[n^{2}+\frac{1}{2} \varepsilon\left(s-\frac{1}{2}\right)^{2}\right], \quad k(s)=-\varepsilon,
$$

where $\varepsilon=1 / n^{2}$. Following Irvine and Caughey [8], one can neglect the inertia terms on the right side of equation (9) on the assumption that the cable stretches in a quasi-static fashion. In essence, small extensions of the cable centerline are assumed to propagate instantaneously along the cable and reduce the dynamic component of cable tension to a function of time alone. This assumption remains valid for low order cable modes [8]. Upon using equation (16) and (17), equation (9) becomes, to first order,

$$
\left(u_{1, s}+\varepsilon u_{2}\right)_{, 3}=0
$$

where it is noted that $v_{i}^{2} / v_{i}^{2}=P_{0} / E A^{i}$ is of order $\varepsilon$. Integration of equation (18) with $u_{1}(0, t)=u_{1}(1, t)=0$ yields

$$
u_{1}(s, t)=f(t) s-\varepsilon \int_{0}^{u_{2}} u_{2}(\eta, t) \mathrm{d} \eta
$$

where

$$
f(t)=\varepsilon \int_{0}^{1} u_{2}(\eta, t) \mathrm{d} \eta
$$

Equations (19) and (20) show that $u_{1}$ is of order $\varepsilon$. Substituting equations (16), (17), (19), and (20) into equations (10) and (11) and neglecting terms of order $\varepsilon$ or less produces

$$
-n^{2} u_{2, s s}-\frac{1}{\mu n^{2}} \int_{0}^{1} u_{2}(\eta) \mathrm{d} \eta=u_{2, t t}+2 v_{c} u_{2, s t} \quad \text { and } \quad-n^{2} u_{3, s s}=u_{3, u}+2 v_{c} u_{3, s t}
$$

where the parameter $\mu=\left|m^{2}\right| / v_{l}^{2}=n^{2} / v_{l}^{2}$ was introduced by Simpson [4].

Equations (21) and (22) represent first order approximations to the equations of motion for a shallow cable arch with level support eyelets. Using $u_{2}=u \mathrm{e}^{i \omega t}$ in (21) results in the following eigenvalue problem governing free in-plane response:

$$
-n^{2} u^{\prime \prime}-\mathrm{i} 2 \omega v_{c} u^{\prime}+\omega^{2} u=C,
$$

where

$$
C=\frac{1}{\mu n^{2}} \int_{0}^{1} u(\eta) \mathrm{d} \eta
$$

with the boundary conditions

$$
\mathrm{u}(0)=u(1)=0 .
$$

Exact analysis of equations (23)-(25) provides the eigenvalues $\omega$ which govern the stability of free linear in-plane motion. The eigenvalues governing the stability of out-of-plane motion are also determined from equations (23)-(25) by noting that the in-plane problem (21) reduces to the out-of-plane problem (22) in the limit $\mu \rightarrow \infty$. The out-of-plane problem is identical to the classical threadline problem [2], except here the coefficient $-n^{2}=v_{t}^{2}-v_{c}^{2}$ 
is negative and the parameter $v_{t}=\left[v_{c}^{2}-n^{2}\right]^{1 / 2}$ may be real or imaginary. The general solution of equation (23) is

$$
u(s)=\mathrm{e}^{\mathrm{i} a s}\left[A \mathrm{e}^{\mathrm{i} b s}+B \mathrm{e}^{-\mathrm{i} b s}\right]+C / \omega^{2},
$$

where

$$
a=-\omega v_{c} / n^{2} \quad \text { and } \quad b=\omega v_{t} / n^{2} .
$$

Upon application of the boundary conditions, equation (26) becomes

$$
u(s)=\frac{C}{\omega^{2}}\left\{\frac{\mathrm{i}^{-\mathrm{i} a}}{2 \sin (b)}\left[1-\mathrm{e}^{\mathrm{i}(a-b)}\right]\left[\mathrm{e}^{\mathrm{i}(a+b) s}-\mathrm{e}^{\mathrm{i}(a-b) s}\right]+\left[1-\mathrm{e}^{\mathrm{i}(a-b) s}\right]\right\} .
$$

Evaluation of expression (24) by using expression (29) and simplification results in the following characteristic equation for $\omega$ :

$$
\omega \sin \left(\omega v_{t} / n^{2}\right)=\frac{2 v_{t}\left[\cos \left(\omega v_{t} / n^{2}\right)-\cos \left(\omega v_{c} / n^{2}\right)\right]}{1-\omega^{2} \mu n^{2}} .
$$

Note that for a minimum catenary, the constant $-n^{2}=m^{2}=v_{t}^{2}-v_{c}^{2}>0$ and $v_{t}$ is therefore real. In this case, the above characteristic equation reduces to Simpson's equation (44) [4] and provides the in-plane natural frequencies of a shallow translating sagged catenary. If, in addition, $v_{c}^{2}=0$, the characteristic equation reduces to that obtained by Irvine and Caughey; see equation (19) of reference [8]. Higher order terms are included in the asymptotic analysis of Triantafyllou [5] which remains valid for translating sagged catenaries having inclined supports.

\section{STABILITY OF OUT-OF-PLANE MOTION}

Letting $\mu \rightarrow \infty$ in equation (30) yields the characteristic equation associated with free linear out-of-plane vibration,

$$
\sin \left(\omega v_{l} / n^{2}\right)=0
$$

with roots

$$
\omega_{k}=k \pi n / \sqrt{\left(v_{c} / n\right)^{2}-1}, \quad k=1,2,3, \ldots
$$

The eigenvalues are imaginary for translation speeds $v_{c} / n<1$ and indicate instability for all out-of-plane vibration modes. As $v_{c} / n$ passes through $v_{c} / n=1$, the eigenvalues approach infinite imaginary values, become real, and then attain finite real values. Thus, all out-of-plane modes become stable for $v_{c} / n>1$, and the transition from instability to stability occurs at the singularity, $v_{c} / n=1$. It is noteworthy that the leading order terms of expression (16) give $v_{t}^{2} p=v_{t}^{2}$ and show that, for the approximate model, $v_{t}^{2} p>0$ when $v_{t}^{2}=n^{2}\left[\left(v_{c} / n\right)^{2}-1\right]>0$. Thus, the out-of-plane modes become stable precisely when the cable becomes tensioned. This result is also seen by inspection of the partial differential equation of motion (22) which undergoes fundamental changes as the translation speed is increased. Equation (22) is elliptic for $v_{t}^{2}<0$, parabolic for $v_{t}^{2}=0$, and hyperbolic for $v_{t}^{2}>0$. Traveling wave solutions to equation (22) exist only in the hyperbolic regime and lead to the stable oscillations predicted by the above eigenvalue problem. A small correction would be expected for the complete model (11) in which one uses the exact expressions (6) and (7) for the cable tension and curvature. In this case, the entire cable is in tension (equation (11) is hyperbolic) for translation speeds $v_{c} / n>\left[1+\left(1 / 4 n^{4}\right)\right]^{1 / 4}$ and is in tension only in the interval $\left|s-\frac{1}{2}\right|<n^{2}\left[\left(v_{c}^{2} / n^{2}\right)^{2}-1\right]^{1 / 2}$ for speeds $1<v_{c} / n<\left[1+\left(1 / 4 n^{4}\right)\right]^{1 / 4}$. 


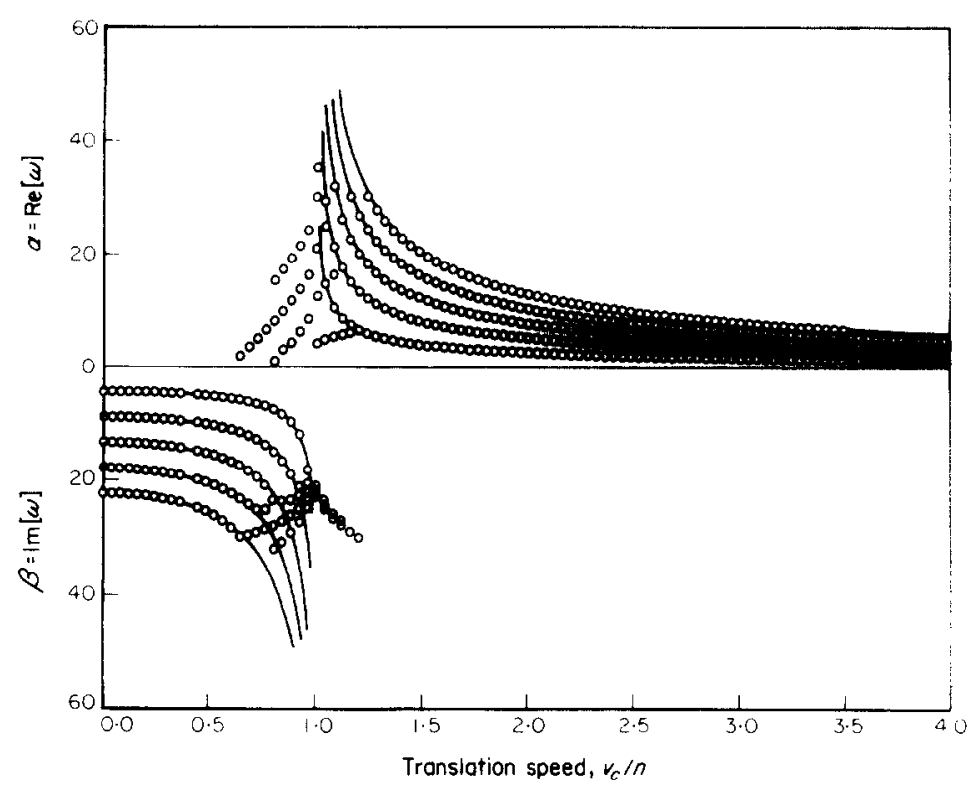

Figure 2. Eigenvalues for out-of-plane modes versus cable translation speed. Modes become stable when $\beta$ vanishes. Solid curves (_) represent the asymptotic solution and dotted curves $(00000)$ denote the numerical solution [7] for the maximum catenary of Figure 3.

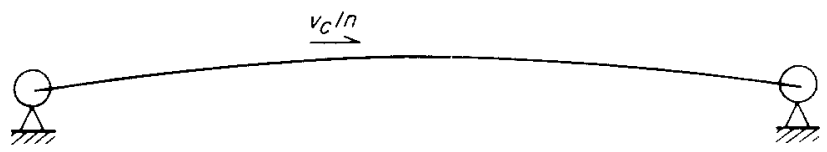

Figure 3. Shallow cable arch. Maximum catenary equilibrium for the case $n^{2}=2$ has an arch height of $6 \cdot 2 \%$ of the cable length.

The solid curves of Figure 2 illustrate the variation of the first five eigenvalues as a function of translation speed over the range $0<v_{c} / n<4$. In this computation, the parameter $n^{2}=2$ and corresponds to the maximum catenary shown in Figure 3, which has an arch height of $6.2 \%$ of the cable length. The numerical solution of reference [7] is also given in Figure 2 (dotted curves) and is based on a Galerkin discretization of the complete model (11). The asymptotic and numerical solutions give almost identical eigenvalues for translation speeds away from $v_{c} / n=1$. In the neighborhood of $v_{c} / n=1$, the indicated complex eigenvalues of the numerical model represent eigenvalues which have not converged, despite the fact that seventy terms were used in the Galerkin approximation. The numerical model converges poorly near $v_{c} / n=1$ due to the singular nature of the eigenvalues in this vicinity.

\section{STABILITY OF IN-PLANE MOTION}

The full characteristic equation (30) is used to obtain the eigenvalues associated with in-plane modes. In this case, the roots of equation (30) are obtained numerically and are not known a priori to be real, imaginary, or complex. The substitution

$$
\omega=\alpha+i \beta
$$


in equation (30) provides the following two equations which are solved simultaneously for $\alpha$ and $\beta$ by using standard Newton-Raphson iteration:

$$
\begin{aligned}
{\left[1-n^{2} \mu\left(\alpha^{2}-\beta^{2}\right)\right]\left\{\alpha\left[\sin \left(\alpha v_{t} / n^{2}\right) \cosh \left(\beta v_{t} / n^{2}\right)\right]-\beta\left[\cos \left(\alpha v_{t} / n^{2}\right) \sinh \left(\beta v_{t} / n^{2}\right)\right]\right\} } \\
+\left[2 \alpha \beta n^{2} \mu\right]\left\{\beta\left[\sin \left(\alpha v_{t} / n^{2}\right) \cosh \left(\beta v_{t} / n^{2}\right)\right]+\alpha\left[\cos \left(\alpha v_{t} / n^{2}\right) \sinh \left(\beta v_{t} / n^{2}\right)\right]\right\} \\
=2 v_{t}\left\{\cos \left(\alpha v_{t} / n^{2}\right) \cosh \left(\beta v_{t} / n^{2}\right)-\cos \left(\alpha v_{c} / n^{2}\right) \cosh \left(\beta v_{c} / n^{2}\right)\right\}
\end{aligned}
$$

and

$$
\begin{aligned}
-\left[2 \alpha \beta n^{2} \mu\right]\left\{\alpha\left[\sin \left(\alpha v_{t} / n^{2}\right) \cosh \left(\beta v_{t} / n^{2}\right)\right]-\beta\left[\cos \left(\alpha v_{t} / n^{2}\right) \sinh \left(\beta v_{t} / n^{2}\right)\right]\right\} \\
+\left[1-n^{2} \mu\left(\alpha^{2}-\beta^{2}\right)\right]\left\{\beta\left[\sin \left(\alpha v_{t} / n^{2}\right) \cosh \left(\beta v_{t} / n^{2}\right)\right]\right. \\
\left.\quad+\alpha\left[\cos \left(\alpha v_{t} / n^{2}\right) \sinh \left(\beta v_{t} / n^{2}\right)\right]\right\} \\
=2 v_{t}\left\{\sin \left(\alpha v_{c} / n^{2}\right) \sinh \left(\beta v_{c} / n^{2}\right)-\sin \left(\alpha v_{t} / n^{2}\right) \sinh \left(\beta v_{t} / n^{2}\right)\right\}
\end{aligned}
$$

For a given index $k$, the roots of equations (34) and (35) are found as $\pm \alpha_{k} \pm \mathrm{i} \beta_{k}$, where $\alpha_{k}$ denotes the natural frequency of the $k$ th mode. In the undamped cable model of the present study, stable modes correspond to those eigenvalues for which $\beta_{k}=0$. Figure 4 depicts the first six eigenvalues computed for the shallow cable arch of Figure 3 . Shown in Figure 4 are the first six values of $\alpha$ and $\beta$ as functions of cable translation speed over the speed range $0<v_{c} / n<4$. Unlike the former out-of-plane problem, the eigenvalues of the in-plane problem can be, and often are, complex. For the stationary arch $\left(v_{c} / n=0\right)$, the eigenvalues are imaginary $\left(\alpha_{k}=0\right)$ and indicate instability for all six in-plane modes. The eigenvalues remain imaginary until adjacent pairs of imaginary loci coalesce as shown by the points marked " $k, k+1 "(k=1,3,5)$ in Figure 4 . At these points, the $k$ th and $(k+1)$ st eigenvalues become complex conjugates and the two modes are still unstable. The eigenvalues remain complex until the final transition marked by " $k$ " $(k=1-6)$ in Figure 4 is reached. Beyond these points, the $k$ th eigenvalue is real $\left(\beta_{k}=0\right)$ and indicates

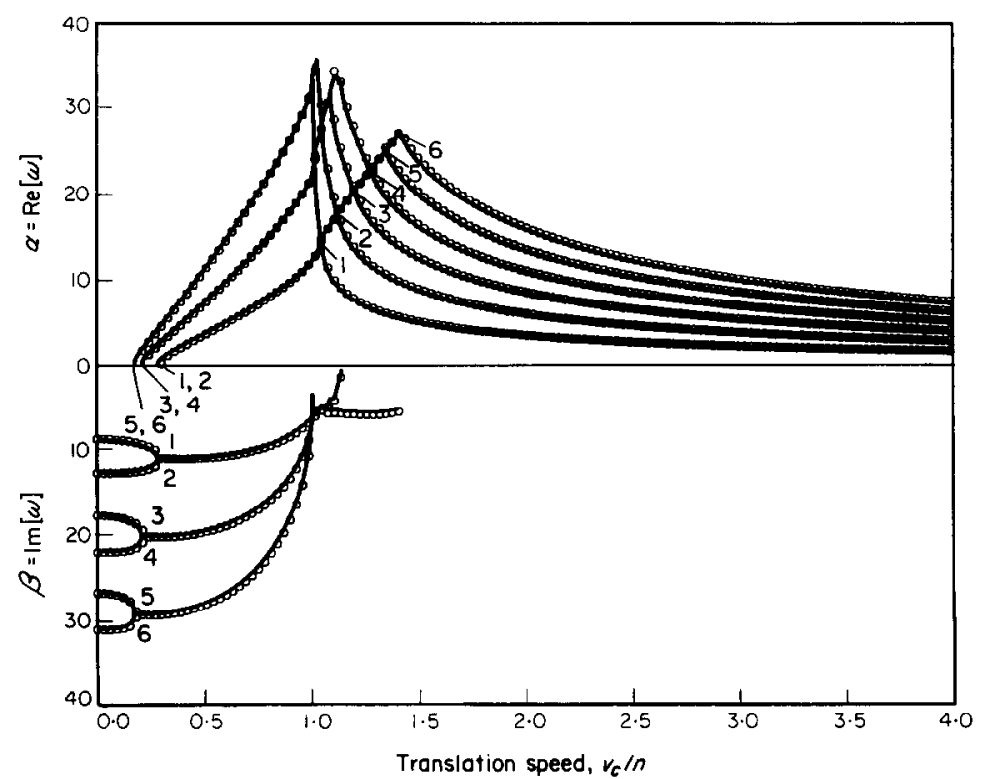

Figure 4. Eigenvalues for in-plane modes versus cable translation speed. Modes become stable when $\beta$ vanishes. Solid curves ) represent the asymptotic solution and dotted curves (०0.00) denote the numerical solution [7] for the maximum catenary of Figure 3. 
stable oscillatory response for the $k$ th in-plane mode. Approximate eigenvalues computed with 70 terms in the Galerkin discretization of the exact equations of motion (9) and (10) [7] are also included in Figure 4 (dotted curves) to illustrate their superb agreement with the asymptotic solution derived here. The stability of the maximum catenary is witnessed by the photograph of Figure 5, reproduced from the experimental study described in references $[7,9]$.

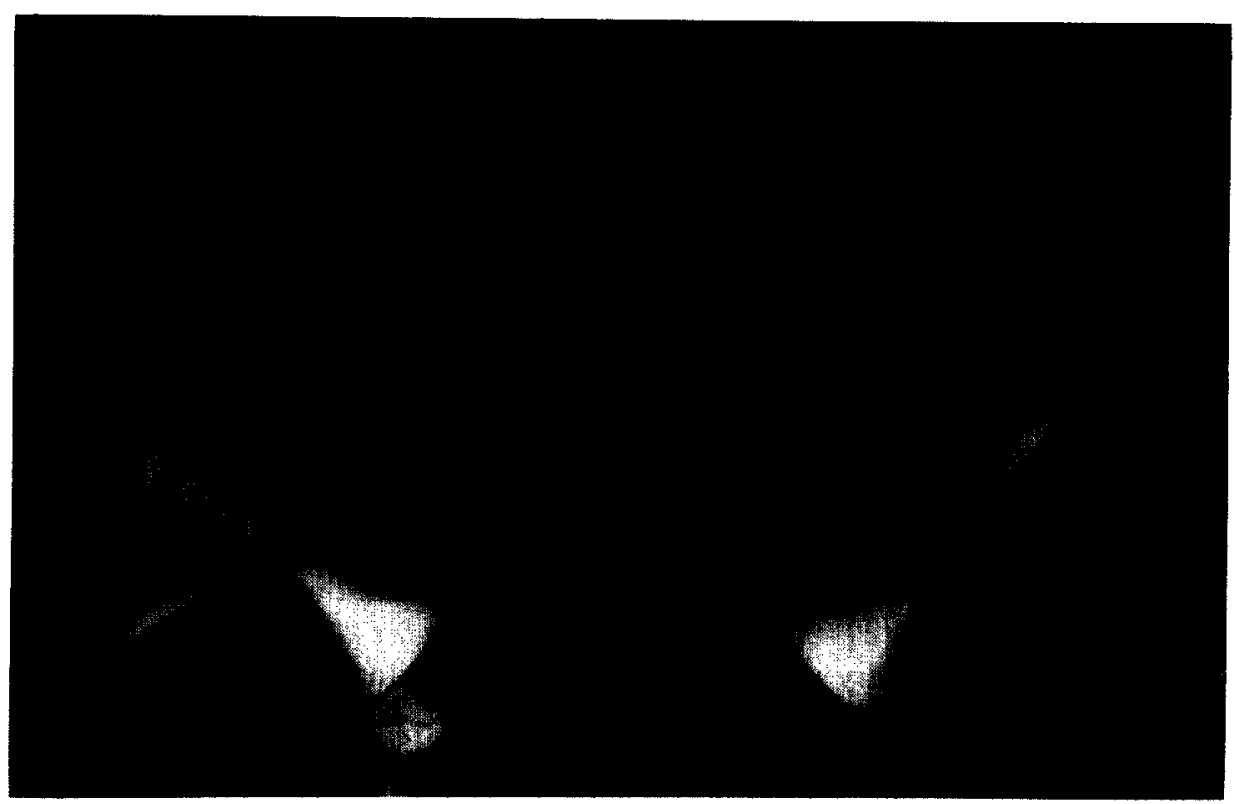

Figure 5. Stable translating cable arch reproduced from [7]. The cable is a $4.8 \mathrm{~mm}$ dia. woven nylon rope of length $1.02 \mathrm{~m}$. Cable circulates counterclockwise with speed $13.8 \mathrm{~m} / \mathrm{s}$, which corresponds to $v / n=8$ for the present catenary with $n^{2}=0 \cdot 3$.

This stability conclusion is not changed upon the introduction of linear damping in the model. As determined in reference [9], the addition of linear viscous damping has a stabilizing effect on all cable modes. In particular, the numerical solution [9] shows that, as the translation speed is increased, the imaginary parts of the eigenvalues $\left(\boldsymbol{\beta}_{k}\right)$ all converge to the same positive value $d / 2$, where $d$ denotes the damping constant in the linear viscous damping model.

\section{SUMMARY AND CONCLUSIONS}

The stability of a translating cable arch (maximum catenary) is analyzed by the exact solution of an approximate model for shallow cable arches. The eigenvalues governing out-of-plane and in-plane stability predict that the cable arch can be stabilized at sufficiently high translation speeds. This fact, which is observed experimentally [7], derives from the speed tensioning capability of the cable equilibrium. The stabilization of out-orplane modes occurs as the associated eigenvalues pass through a singularity during their transition from imaginary to real values. This singularity explains the slow convergence observed in a purely numerical analysis of the exact cable model [7]. Unlike the eigenvalues of the out-of-plane problem which are either real or imaginary, the eigenvalues of the in-plane problem change from imaginary to real values by assuming complex values over specific intervals of the cable translation speed. 


\section{ACKNOWLEDGMENT}

The author gratefully acknowledges the partial support of this research endeavor provided by the Office of Naval Research Young Investigator Program.

\section{REFERENCES}

1. C. D. MOTE, JR. 1972 Shock and Vibration Digest 4(4), 2-11. Dynamic stability of axially moving materials.

2. R. D. SWOPE and W. F. AmEs 1963 Journal of the Franklin Institute 275(1), 36-55. Vibrations of a moving threadline.

3. J. A. WICKERT and C. D. MOTE, JR. 1988 Shock and Vibration Digest 20(5), 3-13. Current research on the vibration and stability of axially moving materials.

4. A. SIMPSON 1972 Journal of Sound and Vibration 20, 177-189. On the oscillatory motions of translating elastic cables.

5. M. S. TRIANTAFYLLOU 1985 Journal of Sound and Vibration 103, 171-182. The dynamics of translating cables.

6. N. C. PeRkins and C. D. Mote, JR. 1987 Journal of Sound and Vibration 114, 325-340. Three dimensional vibration of travelling elastic cables.

7. N. C. PERKINS and C. D. Mote, JR. 1988 Journal of Sound and Vibration 128, 397-410. Theoretical and experimental stability of two translating cable equilibria.

8. H. M. IRVINE and T. K. CAUGHEY 1974 Proceedings of the Royal Society, London A341, 299-315. The linear theory of free vibrations of a suspended cable.

9. N. C. PERKINS 1986 Ph.D. Dissertation, University of California, Berkeley CA. Three dimensional theory and analysis of travelling sagged cable dynamics. 